\title{
Depression and Cardiovascular Disease in Women
}

\author{
Maria Alayde Mendonça Rivera, ${ }^{1,2}$ Ivan Romero Rivera, ${ }^{1,2}$ Walkiria Avila, ${ }^{3}$ Celi Marques-Santos, ${ }^{4}$ (® \\ Francisco Assis Costa, ${ }^{10}$ Carlos Romério Ferro, ${ }^{1}$ Jose Maria Gonçalves Fernandes ${ }^{1}$ \\ Universidade Federal de Alagoas, ${ }^{1}$ Maceió, AL - Brazil \\ Santa Casa de Misericórdia de Maceió, ${ }^{2}$ AL - Brazil \\ Hospital das Clínicas da Faculdade de Medicina da Universidade de São Paulo (HCFMUSP), ${ }^{3}$ São Paulo, SP - Brazil \\ Universidade Tiradentes, ${ }^{4}$ Aracaju, SE - Brazil
}

\section{Abstract}

The prevalence of depression varies from 1 to $17 \%$ in different geographic regions, and its incidence is $70 \%$ higher in women than men. Today, depression affects more than 300 million people worldwide, affecting twice as many women from adolescence to adulthood. In addition to this earlier onset, depression in women tends to be more severe. Cardiovascular disease and depression are chronic diseases that have a major impact on cardiovascular and all-cause morbidity and mortality, with evidence of a two-way relationship between them, in which depression is a predictor of cardiovascular disease and vice versa. In females, the degree of illness and prognosis are more severe when both diseases are present, than when diagnosed alone. In patients with acute or chronic cardiovascular disease, especially women, a systematic screening for depression should be considered as a preventive strategy of cardiovascular events, aiming to reduce the risk of future events. There are still no clinical studies designed to assess the impact of antidepressant treatment on cardiovascular outcomes in women.

\section{Introduction}

Depression is a psychiatric condition characterized by changes in the regulation of mood, behavior, and affection. ${ }^{1}$ It represents a heterogenous group of disease

\section{Keywords}

Cardiovascular Diseases; Coronary Artery Disease, Morbimortality/ prevalence; Women; Risk Factors; Prevention and Control. that share phenotypical features, with different levels of severity. ${ }^{1-4}$

Clinical presentations of depression include: a) mood swings, identified as feelings of sadness, despair, anxiety, emptiness, discouragement or hopelessness, numbness, and a desire to cry. This profound state of unhappiness (dysphoria) may be either transient or be a symptom of a psychopathological syndrome or clinical disorder; b) a syndrome or association of signs and symptoms that include a depressed mood. Depressive syndromes include major depression, minor depression, or dysthymia (persistent depressive disorder); c) accompanied by a psychiatric disorder such as bipolar disorder, schizophrenia, depressive disorder induced by drugs or other medical conditions. ${ }^{1-4}$

Depression is a cause of disability, with high personal, social and economic costs, ${ }^{2}$ requiring a systematic diagnostic investigation and adequate treatment. ${ }^{1-5}$

A prospective study evaluated the relationship of depression and health-related costs in a five-year follow-up of 868 women presenting at the emergency department with suspected acute myocardial infarction (AMI). Depression was associated with up to $53 \%$ increases in cardiovascular costs during the follow-up period. ${ }^{6}$ This association was more evident among women without significant coronary artery disease (CAD), suggesting that depression may cause higher costs in women without conventional markers of cardiac disease. ${ }^{6}$

Depression has high comorbidity rates, similarly to other psychiatric syndromes (anxiety, eating disorders, psychosis), and other diseases (endocrine, respiratory, rheumatological, cardiovascular and degenerative disorders, and cancers). ${ }^{1-4}$ 
The prevalence of depression in different geographic regions worldwide may vary from $1 \%$ to $17 \%$, and its incidence is $70 \%$ higher among women. ${ }^{1-3}$ Approximately $17 \%$ of men and $25 \%$ of women will have an episode of depression throughout life, and $60 \%$ of them will have one or more recurrences. ${ }^{3-5}$

Currently, depression affects more than 300 million people worldwide and, from adolescence onwards, affects twice as many females ${ }^{7}$. In addition to this earlier onset, depression tends to be more severe in women ${ }^{8}$.

Several studies have demonstrated that depression is a risk factor and a prognostic factor of cardiovascular disease (CVD) (especially CAD and cerebrovascular disease) in both sexes, but with particularities related to female sex. ${ }^{8-12}$

The primary objective of this study was to summarize the current knowledge about depression as a risk factor for CVD in women, with emphasis on CAD. Other factors including its prognostic impact in patients with CVD, pathophysiological mechanisms that link depression and CVD, and diagnostic and therapeutic strategies were also evaluated, highlighting possible differences between sexes.

Original articles written in English were searched in PubMed (MEDLINE) and Embase databases from 2010 to 2021, and references of the eligible articles were manually searched. The following descriptors ([MeSH Terms]) were used: "coronary heart disease OR disease, ischemic heart AND (woman) AND (depress*). Inclusion criteria were: a) prospective cohort studies or meta-analysis of prospective cohort studies; b) studies on women or studies presenting analysis by sex; c) studies with participants who did not have CVD in the beginning of the study; d) depression as an outcome of interest; e) AMI or CAD as outcomes of interest; $f$ ) risk estimate (with 95\% confidence interval) of the association between depression and CAD. Study selection and data extraction were performed by two independent investigators; disagreements were resolved by consensus based on the inclusion criteria. Figure 1 depicts the flowchart of study selection.

\section{Depression as a Cardiovascular Risk Factor}

Depression has been recognized as an emerging, nonconventional risk factor for CAD and cerebrovascular disease in both sexes, but with a greater impact on women than men.-12

The risk of cardiovascular disease in women with a diagnosis of depression throughout life is $30-50 \%$ higher in women than men, especially among the young. ${ }^{8}$ Studies have indicated that hormonal changes,

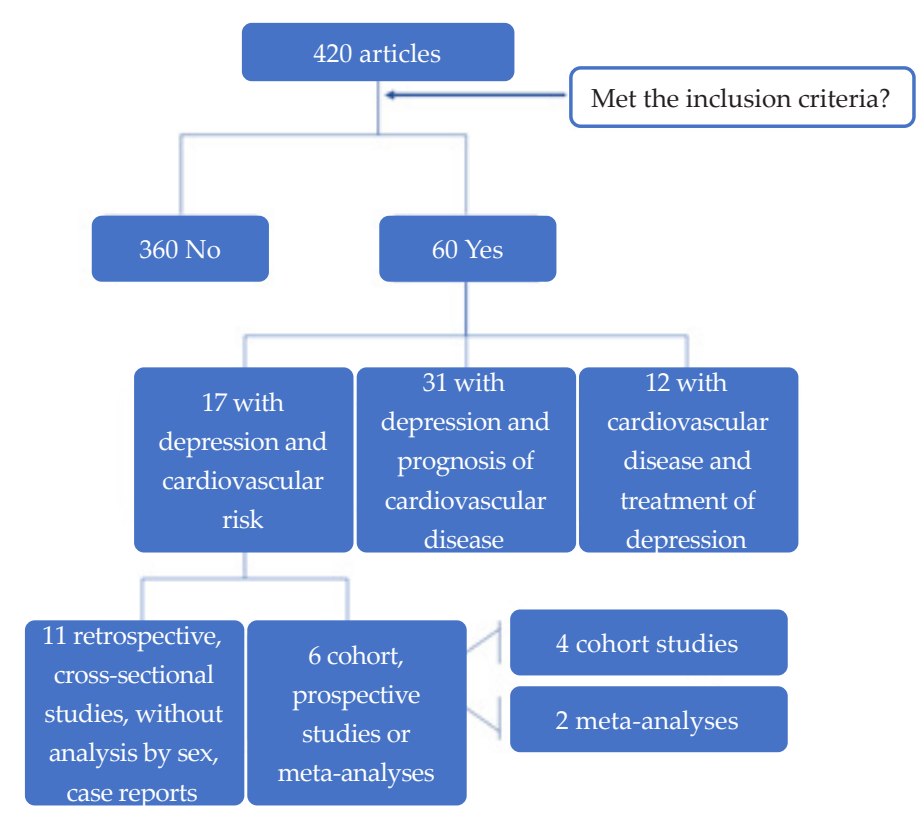

Figure 1 - Flow chart of study selection 
such as menopausal transition, have been associated with a higher risk for the first depressive episode. In addition, depression in perimenopausal women has been associated with a higher frequency of a history mood disorder. ${ }^{2,13}$

A prospective, a 15-year longitudinal study of a representative sample of US adults ( $n=7,641)$, aged 17 to 39 years, showed that depression was associated with an increased risk of death for $\mathrm{CAD}$, with an adjusted hazard ratio of 3.70 (95\% CI, 1.32-10.35) for depression in both men and women, and a 15 -fold adjusted risk of CVD among women (14.57 [95\% CI, 2.65-80.10]). ${ }^{14}$

A nine-year prospective cohort study of 998 women (mean age of 57 years, 28-96 years), without a history of CAD, referred for routine mammography, showed that 185 (18.5\%) were diagnosed with depression at year two; 24 of them $(13.0 \%)$ developed one or more ischemic events by year 10, which was significantly higher than the incidence of $6.5 \%$ in control group $(p<0.001)$. In a logistic regression model, adjusted for other cardiovascular risk factors, including age, depression was the only significant predictive factor for $\mathrm{CAD}$ in women aged less than $<65(\mathrm{OR}=6.56,95 \%$ CI 1.07-40.09, $\mathrm{p}=0.042$ ). The authors drew attention to the finding that in women aged 65 years and over, age was the only significant predictive factor for CAD. ${ }^{15}$

A prospective longitudinal study of 860 women followed for 18 years showed that depression was associated with a twice higher incidence of CAD. ${ }^{16}$

A meta-analysis of 30 prospective cohort studies ( $n=893,850$ and 59,062 cases of CAD), with a follow-up period ranging from two to 37 years, showed a significant, independent association of depression with an increased risk of chronic CAD and AMI in both sexes. ${ }^{17}$

Another meta-analysis on the association between depression, risk of AMI and death for CAD included 19 prospective cohort studies, with a total of 323,709 participants followed for 4-37 years demonstrated that depression was associated with a significantly increased risk of myocardial infarction and coronary mortality in both sexes. ${ }^{18}$

Regarding the important relationship between depression and CAD, it is worth discussing the results of the VIRGO (Variation in Recovery: Role of Gender on Outcomes of Young AMI Patients) Study, ${ }^{19}$ the Women's Health Initiative (WHI) ${ }^{20}$ and the Nurse's Health Study. ${ }^{21}$

The VIRGO Study was an observational study on clinical presentation, treatment and outcomes of 2,000 young women and 1,000 young men (aged from
18 to 55 years old) with AMI, seen at 100 participating hospitals. The study showed that $50 \%$ of women and $25 \%$ of men had a history of depression and, at the moment of the ischemic episode, $39 \%$ of women and $29 \%$ of men had depression. ${ }^{19}$

Data from the WHI Observational Study, ${ }^{20}$ which followed up 93,676 postmenopausal women for an average of 4.1 years, demonstrated that depression was significantly related to CVD risk (odds ratio of 1.60 for cerebrovascular disease or angina). In women without a history of cardiovascular disease, depression was an independent predictor of death for CVD (relative risk, 1.50) and all-cause mortality (relative risk. 1.32) after adjustment for age, race, education, income, diabetes, hypertension, smoking, high cholesterol level requiring medication, body mass index, and physical activity. The authors called attention to the fact that, so far, whether early treatment with antidepressants of patients without a history of CVD will lower the risk for the disease is still unknown. ${ }^{20}$

In a cohort study of 63,469 women without cardiovascular disease, followed for four years, a diagnosis of depression was significantly associated with fatal and nonfatal AMI. ${ }^{18}$ The authors highlighted that sudden cardiac death in depressive women may be more associated with sympathetic nervous system activation, higher resting heart rate and greater use of antidepressants. ${ }^{21}$

Based on the large amount of evidence supporting that depression is an independent risk factor for CVD in men and especially in women, ${ }^{22}$ it has been included in many guidelines on CVD prevention..$^{8-12,22,23}$ However, depression has not been included in any of the current cardiovascular risk scores. ${ }^{11,12,23}$

\section{Depression as a Prognostic Factor in Cardiovascular Disease Patients}

Depression has also been regarded as a predictor of worse prognosis in men and women diagnosed with AMI, unstable angina, and stroke, and those with angiographic confirmation of chronic CAD. ${ }^{8-11,24}$

Epidemiological studies have reported a higher prevalence of depression in patients with CVD than in general population and that individuals with depression are more likely to develop AMI, heart failure and stroke. ${ }^{8-10}$

Depression is two to three times more prevalent in CAD patients (up to $30 \%$ in this population) and two times higher in women than men. ${ }^{8}$ 
In a prospective study of patients with AMI, the diagnosis of depression was more frequent in women than in men; women with depression had a two-fold higher rate of complications such as reinfarction, recurrent ischemia, cardiogenic shock, cardio-respiratory arrest and in-hospital death. ${ }^{25}$

In post-acute myocardial infarction patients, a diagnosis of depression increased the risk for recurrent cardiovascular event within two years. ${ }^{25}$

In a post-hoc analysis of a randomized, double-blind study (Darapladib vs. placebo) of 15,828 patients with chronic CAD (19\% women), followed for a median of 3.7 years, women with depression had a higher risk of infarction, stroke, and cardiovascular death. ${ }^{26}$

In patients with stroke, depression is associated with higher mortality and recurrence rates. As compared with men, women with stroke are twice more likely to have depression, and early onset of treatment has a positive impact on physical and cognitive recovery in these cases. ${ }^{24}$

A meta-analysis of eight studies, involving 125,763 patients, demonstrated a prevalence of $21.5 \%$ of depression in patients with heart failure. The presence of depression was associated with a worse prognosis and higher mortality, especially in older patients and in those taking antidepressants, with no difference between sexes. ${ }^{27}$ The authors discussed the importance of a tailored antidepressant prescription to this group of patients, considering symptoms, and severity of heart failure, depression and other conditions, ${ }^{27}$ since treatment with antidepressant may contribute to higher mortality in these patients.

In addition to the above, in general, depression also contributes to a poorer adherence to treatment, self-care practices and rehabilitation programs, ${ }^{16-19}$ with a negative impact especially on women. In the presence of CAD and/or cerebrovascular disease, depression is associated with persistence of symptoms, poorer quality of life, early and frequent readmissions and higher mortality rates in women. ${ }^{16-19}$

These data support the need for a systematic investigation of depression in patients with acute and chronic CVDs, considering the frequent association between these diseases and its potential devastating effect, on these individuals, particularly on women. ${ }^{16-19}$

\section{Mechanisms that Link Depression to Cardiovascular Diseases}

Depression and CVD are chronic diseases that have a great impact on cardiovascular morbidity and mortality.
Evidence supports a two-way relationship between these conditions, in which depression is a predictor of cardiovascular disease and vice versa. ${ }^{28}$ In women, the combination of depression and CVD leads to more severe disease and a poorer prognosis, than when presented alone. ${ }^{29}$

However, despite increasing data supporting that both depression and CVD share etiological pathways related to the immune system and inflammation, studies to elucidate this association and how women are more affected during hormonal changes related to reproductive events (menstrual cycle, pregnancy, menopause) are still needed. ${ }^{5,24,28-30}$

The current hypothesis is that in women, the low-grade chronic inflammation contributes to the development of both depression and CVD, when associated to dysregulation of some biological systems, such as the hypothalamic-pituitary-adrenal axis, the reninangiotensin-aldosterone system, and the serotoninkynurenine pathway. ${ }^{5,24,28-30}$ Dysregulation of these systems is accompanied by increases in inflammatory markers, endothelial dysfunction, platelet activation and coagulation, as well as increase in sympathetic tone. ${ }^{28-31}$ These changes, combined with traditional and/or emerging cardiovascular risk factors and reproductive hormonal fluctuations, may generate an even worse inflammatory response that contributes to the development or aggravation of the depression-CVD relationship. ${ }^{29-31}$

In a cohort study, ${ }^{32}$ where participants were followed from birth to the age of 18 years, individuals with at least one episode of depression had higher levels of C-reactive protein and triglycerides, greater insulin resistance and a higher mass index corporal, and this association was more frequent in female participants. ${ }^{32}$ These findings corroborate the hypothesis of the association between depression, inflammation, and cardiovascular risk. 5,28-30,32

The pattern of inflammatory response in women may vary over the years according to the exposure to endogenous sex hormones (estrogens and progesterone), especially estradiol (one of the endogenous estrogens), which seems to be more directly associated with suppression of inflammatory cytokines (interleukin-6 and TNF-alpha) and increase of anti-inflammatory cytokine production (interleukin-10). Regarding plasma levels, high and low levels of estradiol are associated with an anti-inflammatory and a pro-inflammatory response, respectively, while progesterone has predominantly anti-inflammatory effects. ${ }^{28}$ 
Thus, in women, hormonal variations throughout life can have a great impact on their cardiovascular health and contribute to the developing of mood disorders. Therefore, menarche, early menopause and peri-menopause are periods that increase the risk of developing of CVD, while the perinatal period and menopause increase the chance of both occurring in association. ${ }^{28}$

With respect to the use of exogenous sex hormones for contraceptive purposes, relief of menstruation-related abdominal pain, and treatment of polycystic ovary syndrome and endometriosis, there is still little evidence supporting that it may increase the risk for depression and CVDs. ${ }^{28}$ However, it is worth to mention that the use of oral contraceptives like estrogen plus progesterone may raise the risk of systemic arterial hypertension, thromboembolism, and ischemic stroke. ${ }^{28}$

\section{Diagnosis}

All professionals involved in the management of CVD patients must be aware of this important and close relationship between depression and CVD, considering the potential role of the former in favoring the development and worsening the prognosis of the latter. $^{8-21}$ In addition, these healthcare providers must recognize the worse impact of this association among women. ${ }^{7,16-19}$

Similar to other diseases, the diagnosis of depression initially depends on clinical history and physical examination, with history being the most important component of this assessment. This should be taken from the patient and complemented by information given by family members and other physicians, with patient's consent and respecting the confidentiality of the information obtained. ${ }^{1,3,4}$

Symptoms of depression include persistent feeling of sadness, loss of interest or pleasure in daily activities, insomnia or hypersomnia, significant weight gain or loss ( $5 \%$ or more within a month), decrease or increase in appetite, fatigue or low energy, decreased capacity to concentrate or to make decisions, sense of uselessness, inappropriate or excessive guilt, frequent thoughts of death, and suicide ideation or attempt. ${ }^{1,3,4}$

Psychomotor retardation (slowed speech, decreased speech production, slowed movement) or agitation (restlessness, wringing of hands, inability to keep still, pulling clothes or skin) can be identified during physical examination, which can be confirmed by other people over time. ${ }^{1,3,4}$
The assessment of a person with depression should include the analysis of the chronology of depressive symptoms - current symptoms, history of depression events, the course and treatment of disease - the impact of depressive episodes on occupational and interpersonal functioning, and presence of attenuating or aggravating factors (life-stressing events, and social or occupational circumstances), psychiatric diseases and other concomitant conditions. ${ }^{1-4}$

In patients with acute or chronic CVDs, the screening for depression should be considered as a preventive strategy against cardiovascular events.-10 This investigation should be performed during hospitalization and at regular intervals thereafter. ${ }^{33}$

This recommendation for the investigation of depression in CVD patients has been progressively included in guidelines on primary and secondary cardiovascular prevention, calling attention to specificities of depression in women with CVDs. ${ }^{8,9,11,}$ $21,22,31,33$

There are several diagnostic tools for depression, with good sensitivity, specificity and negative predictive value, that can be used in clinical practice. . $^{8,31,33}$ Application of these instruments requires specific training and should be done by experienced professionals, with the supervision of a psychiatrist. ${ }^{8}$ However, there are self-answered questionnaires that have been validated and used in several studies, and used in the screening and diagnostic confirmation of depression in patients with CVDs. ${ }^{25,31}$

In this regard, we recommend the Patient Health Questionnaire (PHQ)-2 (which contains two questions on depression) as a first-step approach, and patients who screen positive should be further evaluated with the nine-question "Patient Health Questionnaire" (PHQ)-9 (with nine questions) for confirmation of the diagnosis. $8,10,11,22,31,33$ These questionnaires have a sensitivity of $96 \%$ and specificity of $71 \%$ (PHQ-2) and $72 \%$ (PHQ-9), ${ }^{31}$ and can be freely accessed at https://bit. ly/2VvPHIG (PHQ-2) e https://bit.ly/2PY3INz (PHQ-9). ${ }^{31}$

\section{Treatment}

Except for heart failure, ${ }^{27}$ there is evidence suggesting that treatment of depression in CVD patients improves depressive symptoms, adherence to other therapies and tends to reduce depressive events, although it has no effect on reducing cardiovascular mortality and all-cause mortality. ${ }^{8-11,21-23,31,33,34}$ 
In patients with CVDs, several therapeutic interventions for depression have been investigated in observational studies and clinical trials and shown to be effective either alone or in combination - psychotherapy, psychodynamic therapy, behavioral cognitive therapy, stress management, use of antidepressants, electroconvulsive therapy, and exercises. . $, 8,33,34^{-1}$

The benefits of the treatment of depression to the course of patients with CVD seem to be greater when there is an association of psychotherapy, especially cognitive behavioral therapy, with antidepressant drugs..$^{8,31,33}$ These data reinforce the need for a multiprofessional and multidisciplinary intervention that may include, in addition to a cardiologist, a psychiatrist, a psychologist, a physical educator, among others. ${ }^{23}$

Different psychotherapeutic approaches help depressed individuals understand how their ideas, feelings and behaviors contribute to worsening of depression, and provide them with coping strategies that may help these patients retake control of themselves. ${ }^{8,23}$ When used alone, psychotherapy is as effective as antidepressant drugs in improving depressive symptoms, but several reports have shown that better results are achieved when these both strategies are used in combination. ${ }^{8}$

In addition, physical exercise, especially aerobic exercise seems effective in the treatment of depression and may be complementary to the effect of antidepressants in patients considered "poor responders" to these medications. ${ }^{8}$

The currently available antidepressants for the treatment of depression include selective inhibitors of serotonin uptake (fluoxetine, sertraline, paroxetine, fluvoxamine, citalopram, escitalopram), inhibitors of serotonin and norepinephrine uptake (desvenlafaxine, duloxetine, levomilnacipran, milnacipran, venlafaxine), and antidepressants with new mechanisms of action (bupropion, mirtazapine, mianserin, nefazodone, trazodone). ${ }^{8,31,33,34}$

Four randomized clinical trials evaluated the effect of antidepressants (bupropion, citalopram, escitalopram, mirtazapine, sertraline e venlafaxine) versus conventional treatment in post-AMI patients diagnosed with depression. ${ }^{31}$ Three studies, involving 2,091 patients, have shown a significant improvement of depressive symptoms with treatment during a follow-up of six to 30 months. However, in a study with 331 patients followed for 18 months, no difference was found in depressive symptoms between the groups. ${ }^{31}$ None of the studies demonstrated that the treatment of depression was effective in reducing events, hospitalization, and all-cause and cardiovascular mortality, after a mean follow-up of 16 months. ${ }^{28}$ One study showed that the use of antidepressant resulted in better symptom control in women than men. ${ }^{31}$ None of the studies reported adverse effects of the therapy used.

A randomized clinical trial ${ }^{34}$ with 300 post-AMI patients with depression (39.3\% were women), investigated the use of escitalopram vs. placebo for 24 months and showed a significant reduction in reinfarction during a follow-up period of eight years, with no significant difference in cardiovascular and all-cause mortality.

Levine et al., ${ }^{35}$ pointed out that the analysis of data of numerous studies conducted with depressed patients after hospitalization for acute CAD has revealed that the failure of treatment with antidepressants is associated with higher risk of mortality and cardiovascular events in the follow-up of these patients. ${ }^{35}$

It is important to mention that fluoxetine and paroxetine should not be administered to patients with breast cancer, due to their inhibitory effect on tamoxifen. ${ }^{21}$

In addition, tricyclic antidepressants should be avoided in patients with structural CVD because of their effects on cardiac conduction, seen in the electrocardiogram as prolongation of the PR interval, QRS complex and QT interval, and T-wave flattening. ${ }^{8,27,31}$ These effects increase the likelihood of malignant ventricular arrhythmias and sudden cardiac death, mainly in women and older patients with conduction disturbances, heart failure, or chronic or acute CAD. . $^{87,31}$

So far, there is no study evaluating the impact of antidepressant treatment on cardiovascular outcomes in women. ${ }^{23}$

Table 1 describes the currently used drugs for the treatment with depression in patients with CVD, including daily doses, main side effects and impact on QT interval.

Electroconvulsive therapy, which has a successful rate of approximately $80 \%$, and should be used as the last therapeutic option, after failure of other treatments. It also should be postponed in patients with hemodynamic instability and new-onset, still uncontrolled, arterial hypertension, to minimize the risk of complications (persistent hypertension, ischemia, arrythmias, prolonged asystole, and heart failure). ${ }^{8}$

Due to the frequent association between menopause, CVD and depression, it is important to stress out that hormone therapy should not be used for primary 
Table 1 - Currently used drugs in the treatment of depression in patients with cardiovascular diseases

\begin{tabular}{ccc}
\hline DRUG & $\begin{array}{c}\text { DAILY DOSE } \\
(\mathrm{mg})\end{array}$ & $\begin{array}{c}\text { EFFECT ON QT } \\
\text { INTERVAL }\end{array}$ \\
\hline
\end{tabular}

INIBIDORES SELETIVOS DA RECEPTAÇÃO DA SEROTONINA

Citalopram
$\begin{aligned} & 20-40 \\ & \text { Escitalopram }\end{aligned}$

INHIBITORS OF SEROTONIN AND NOREPINEPHRINE UPTAKE

$\begin{array}{llll}\text { Desvenlafaxine } & 50-100 & \text { None } & \text { Insomnia (VL), sexual dysfunction (VL) } \\ \text { Venlafaxine } & 75-375 & \text { Little } & \text { Insomnia (VL), sexual dysfunction (M) } \\ \text { Duloxetin } & 60-120 & \text { None } & \text { Insomnia (VL), sexual dysfunction (VL) }\end{array}$

SELECTIVE INHIBITORS OF SEROTONIN-DOPAMINE UPTAKE

$\begin{array}{llll}\text { Bupropion } & 300 & \text { Little } & \text { Insomnia (L) }\end{array}$

ALPHA-1 RECEPTOR ANTAGONISTS

$\begin{array}{lccc}\text { Mirtazapine } & 15-45 & \text { Little } & \text { Wight gain }(\mathrm{H}) \text {, sexual dysfunction (VL) } \\ \text { Mianserin } & 60-120 & \text { Little } & \text { Weight gain (H), sexual dysfunction (VL) }\end{array}$

SEROTONIN MODULATORS

$\begin{array}{lccc}\text { Nefazodone } & 300-600 & \text { None } & \text { orthostatic hypotension } \\ \text { Trazodone } & 200-500 & \text { Little } & \text { orthostatic hypotension (VL or M), Weight gain (VL), sexual } \\ \text { dysfunction (VL) }\end{array}$

*Side effect degree: H: high; M: medium; L: low; VL: very low

or secondary prevention of CVD or treatment of depression. ${ }^{2,9,21}$

\section{Final Considerations}

So far, scientific evidence has indicated the presence of common biomarkers between depression and CVD, which determines a worse prognosis for patients with both conditions, especially among women. Considering that depression is more prevalent in women and that depressed women are more likely to have cardiovascular risk factors and more severe forms of CVD than men, a systematic screening for depression and its adequate treatment in women with suspected or confirmed CVD is warranted. Besides, randomized, clinical trials to investigate the impact of treatment of depression on cardiovascular outcomes in men and women with CVD are urgently needed. 


\section{Author contributions}

Conception and design of the research: Rivera MAM, Rivera IR. Acquisition of data: Rivera MAM, Rivera IR, Avila W, Marques-Santos C, Costa FA, Ferro $\mathrm{CR}$, Fernandes JMG. Analysis and interpretation of the data: Rivera MAM, Rivera IR, Avila W, MarquesSantos C, Costa FA, Ferro CR, Fernandes JMG. Writing of the manuscript: Rivera MAM, Rivera IR. Critical revision of the manuscript for intellectual content: Rivera MAM, Rivera IR, Avila W, Marques-Santos C, Costa FA, Ferro CR, Fernandes JMG. Maria Alayde Mendonça Rivera MAM, Ivan Romero Rivera IR, Walkiria Avila W, Celi Marques-Santos C, Francisco
Assis Costa FA, Carlos Romério Ferro CR, Jose Maria Gonçalves Fernandes JMG.

\section{Potential Conflict of Interest}

No potential conflict of interest relevant to this article was reported.

\section{Sources of Funding}

There were no external funding sources for this study.

\section{Study Association}

This study is not associated with any thesis or dissertation work.

\section{References}

1. American Psychiatric Association. Diagnostic and statistical manual of mental disorders (DSM-5). 5th ed Washington, D.C.;2013.

2. Stute P, Spyropolou A, Karageorgiou V, Cano A, Bitzer J, Ceausu J et al. Management of depressive symptoms in peri- and postmenopausal women: EMAS position statement. Maturitas. 2020; 131;91-101. Doi:10.1016/i.maturitas.2019.11.002

3. Mitchell AJ, Vaze A, Rao S. Clinical diagnosis of depression in primary care: a meta-analysis. Lancet. 2009; 374(9690):600-19

4. McAllister-Williams RH, Arango C, Blier P, Demyttenaere K, Falka $\mathrm{P}$, Gorwoodg $\mathrm{P}$ et al. The identification, assessment and managemen of difficult-to-treat depression: An international consensus statement J Affect Disord.2020;267-82. Doi: 10.1016/j.jad.2080.023.

5. Slavich GM, Sacher J. Stress, sex hormones, inflammation, and major depressive disorder: Extending Social Signal Transduction Theory of Depression to account for sex differences in mood disorders. Psychopharmacology. 2019;236(10):3063-79.

6. Rutledge T, Vaccarino V, Johnson D, Bittner V, Olson MB, Linke SE, et al. Depression and Cardiovascular Healthcare Costs among Women with Suspected Myocardial Ischemia: Prospective Results from the Women's Ischemia Syndrome Evaluation (WISE). J Am Coll Cardiol 2009;53(2):176-83

7. Salk RH, Hyde JS, Abramson LY. Gender diferences in depression in representative national samples: meta-analyses of diagnoses and symptoms. Psychol Bull. 2017;143(8):783-822.

8. Vaccarino V, Badimon L, Bremner JD, Cenko E, Cubedo J, Dorobantu M, et al. Depression and coronary heart disease: 2018 position paper of the ESC working group on coronary pathophysiology and microcirculation. Eur Heart J. 2019; 0: 1-15.

9. Bushnell C, McCullough LD, Awad IA, Chireau MV, Fedder WN, Furie $\mathrm{KL}$, et al. Guidelines for the Prevention of Stroke in Women: A Statement for Healthcare Professionals From the American Heart Association/ American Stroke Association. Stroke. 2014;45(5):1545-88

10. Lichtman JH, Froelicher ES, Blumenthal JA, Carney RM, Doering LV,Frasure-Smith N, et al. Depression as a Risk Factor for Poor Prognosis Among Patients With Acute Coronary Syndrome: Systematic Review and Recommendations. A Scientific Statement From the American Heart Association. Circulation. 2014;129(12):1350-69.

11. Piepoli MF, Hoes AW, Agewall S, Albus C, Brotons C, Catapano AL, et al. 2016 European Guidelines on cardiovascular disease prevention in clinical practice The Sixth Joint Task Force of the European Society of Cardiology and Other Societies on Cardiovascular Disease Prevention

in Clinical Practice (constituted by representatives of 10 societies and by invited experts). Eur Heart J. 2016;37(29):2315-81.

12. Précoma DB, Oliveira GMM, Simão AF, Dutra OP, Coelho OR, Izar COM, et al. Atualização da Diretriz de Prevenção Cardiovascular da Sociedade Brasileira de Cardiologia - 2019. Arq Bras Cardiol. 2019;113(4):787-891.

13. Soares CN. Depression and Menopause: An Update on Current Knowledge and Clinical Management for this Critical Window. Med Clin North Am. 2019;103(4):651-66.

14. Shah AJ, Veledar E, Hong Y, Bremner JD, Vaccarino V. Depression and history of attempted suicide as risk factors for heart disease mortality in young individuals. Arch Gen Psychiatry. 2011; 68 (11): 1135-42. doi: 10.1001/archgenpsychiatry.2011.125.

15. Jiang X, Asmaro R, O'Sullivan DM, Modi J, Budnik E, Schnatz PF. Depression may be a risk factor for coronary heart disease in midlife women <65 years: A 9-year prospective cohort study. Int J Cardiol. 2018;27:8-12. doi: 10.1016/j.ijcard.2018.05.085. Epub 2018 May 24.

16. Gan Y, Gong Y, Tong X, Sun H, Cong Y, Dong X, et al. Depression and the risk of coronary heart disease: A meta-analysis of prospective cohort studies. BMC Psychiatry. 2014;14:371-8. Doi: 1

17. O'Neil A, Fisher AJ, Kibbey KJ, Jacka FN, Kotowicz MA, Williams LJ et al. Depression is a risk factor for incident coronary heart disease in women: an 18-year longitudinal study. J Affect Disord. 2016;196:117-24. Doi:10.1016/j.jad.2016.02.029.

18. Wu Q, Kling JM. Depression and the risk of myocardial infarction and coronary death: A meta-analysis of prospective cohort studies. Medicine (Baltimore). 2016; 95(6):e2815. doi: 10.1097/MD.0000000000002815.

19. Smolderen KG, Strait KM, Dreyer RP, D'Onofrio G, Zhou S, Lichtman $\mathrm{JH}$, et al. Depressive Symptoms in Younger Women and Men With Acute Myocardial Infarction: Insights From the VIRGO Study. J Am Heart Assoc. 2015;4(4):e001424 doi: 10.1161/JAHA.114.001424.

20. Wassertheil-Smoller S, Shumaker S, Ockene J, Talavera GA, Greenland $\mathrm{P}$, Cochrane B, et al. Depression and Cardiovascular Sequelae in Postmenopausal Women. The Women's Health Initiative (WHI). Arch Intern Med. 2004; 164(3): 289-98

21. Whang W, Kubzansky LD, Kawachi I, Rexrode KM, Kroenke CH, Glynn $\mathrm{RJ}$, et al. Depression and risk of sudden cardiac death and coronary heart disease in women: results from the Nurses' Health Study. J Am Coll Cardiol. 2009;53(11):950-8.

22. Brown HL, Warner JJ, Gianos E, Gulati M, Hill AJ, Hollier M, et al. Promoting Risk Identification and Reduction of Cardiovascular Disease in Women 
Through Collaboration With Obstetricians and Gynecologists: A Presidential Advisory From the American Heart Association and the American College of Obstetricians and Gynecologists. Circulation. 2018;(24):e843-e852.

23. Arnett DK, Blumenthal RS, Albert MA, Buroker AB, Goldberger ZD Hahn EJ, et al. 2019 ACC/AHA Guideline on the primary prevention of cardiovascular disease: a report of the American College of Cardiology/ American Heart Association Task Force on Clinical Practice Guidelines. Circulation. 2019;140(11):e596-e646.. DOI: 10.1161/CIR.0000000000000678

24. Bucciarelli V, Caterino AL, Bianco F, Caputti CG, Salerni S, Sciomer, S et al. Depression and cardiovascular disease: The Deep blue sea of women's heart. Trends in Cardiovasc Med. 2020;30(3):170-6.

25. AbuRuz ME, Al-Dweik G. Depressive Symptoms and Complications Early after Acute Myocardial Infarction: Gender Differences. Open Nurs J. 2018;12:205-14. Doi: 10.2174/187443460181.2010 205

26. Guimarães PO, Granger CB, Stebbins A, Chiswell K, Held C, Hochman JS et al. Sex Differences in Clinical Characteristics, Psychosocial Factors, and Outcomes Among Patients With Stable Coronary Heart Disease: Insights from the STABILITY (Stabilization of Atherosclerotic Plaque by Initiation of Darapladib Therapy) Trial. J Am Heart Assoc. 2017;6(9):1-16.

27. He W, Zhou Y, Ma J, Wei B, Fu W. Effect of antidepressants on death in patients with heart failure: a systematic review and meta-analysis. Heart Fail Rev. 2020;25(6):919-26

28. Mattina GF, Van Lieshout RJ, Steiner M. Infammation, depression and cardiovascular disease in women: the role of the immune system across critical reproductive events. Ther Adv Cardiovas Dis. 2019;13:1753944719851950.
29. Mingjing S, Xiaodong L, Deguo J, Hongjun T, Yong X, Lina W et al Depression and cardiovascular disease: Shared molecular mechanisms and clinical implications. Psychiat Res. 2020;285: 112802. Doi: 10.1016/j. psyChres.2020.112.802

30. Kahl KG, Stapel B, Frieling H. Link between depression and cardiovascular diseases due to epigenomics and proteomics: Focus on energy metabolism. Progr Neuropsychopharmacol Biol Psychiatry. 2019;89:146-57. Doi: 10.1016/j.psychres.2020.112.802

31. Frost J, Rich Jr RL, Robbins CW, Stevermer JJ, Chow RT, Leon KK, et al. Depression following Acute Coronary Syndrome events: Screening and Treatment Guidelines from the AAFP. Am Fam Physician. 2019;99(12):786A-786J

32. Perry BI, Oltean BP, Jones PB, Khandaker GM. Cardiometabolic risk in young adults with depression and evidence of inflammation: A birth cohort study. Psychoneuroendocrinology. 2020;116:104682.

33. Jha MK, Qamar A, Vaduganathan M, Charney DS, Murrough JW. Screening and Management of depression in patients with cardiovascular disease. JACC State-of-the-art-review. Jam Coll Cardiol. 2019;73(14):1827-45.

34. Kim JM, Stewart R, Lee YS, Lee HJ, Kim MC, Kim JW, et al. Effect of Escitalopram vs Placebo Treatment for Depression on Long-term Cardiac Outcomes in Patients With Acute Coronary Syndrome A Randomized Clinical Trial. JAMA. 2018; 320 (4):350-7.

35. Levine GN, Cohen BE, Commodore-Mensah Y, Fleury J, Huffman JC, Khalid U, et al. Psychological Health, Well-Being, and the Mind-HeartBody Connection: A Scientific Statement From the American Heart Association. Circulation. 2021;143(10): e763-e783. 